Ferrata Storti Foundation

\title{
MicroRNA-21 maintains hematopoietic stem cell homeostasis through sustaining the nuclear factor- $\kappa B$ signaling pathway in mice
}

\author{
Mengjia Hu, Yukai Lu, Hao Zeng, Zihao Zhang, Shilei Chen, Yan Qi, Yang Xu, \\ Fang Chen, Yong Tang, Mo Chen, Changhong Du, Mingqiang Shen, Fengchao \\ Wang, Yongping Su, Song Wang" and Junping Wang\#
}

Haematologica 2021

Volume 106(2):412-423

\section{Correspondence:}

JUNPING WANG

wangjunping@tmmu.edu.cn

Received: August 30, 2019.

Accepted: January 20, 2020.

Pre-published: January 23, 2020.

https://doi.org/10.3324/haematol.2019.236927

(C)2021 Ferrata Storti Foundation

Material published in Haematologica is covered by copyright. All rights are reserved to the Ferrata Storti Foundation. Use of published material is allowed under the following terms and conditions:

https://creativecommons.org/licenses/by-nc/4.0/legalcode. Copies of published material are allowed for personal or internal use. Sharing published material for non-commercial purposes is subject to the following conditions:

https://creativecommons.org/licenses/by-nc/4.0/leǵalcode, sect. 3. Reproducing and sharing published material for commercial purposes is not allowed without permission in writing from the publisher.
${ }^{1}$ State Key Laboratory of Trauma, Burns and Combined Injury, Institute of Combined Injury, Chongqing Engineering Research Center for Nanomedicine, College of Preventive Medicine, Third Military Medical University, Chongqing, China

"SW and JW contributed equally as co-senior authors

\section{ABSTRACT}

L ong-term hematopoietic output is dependent on hematopoietic stem cell (HSC) homeostasis which is maintained by a complex molecular network in which microRNA play crucial roles, although the underlying molecular basis has not been fully elucidated. Here we show that microRNA-21 (miR-21) is enriched in murine HSC, and that mice with conditional knockout of miR-21 exhibit an obvious perturbation in hematopoiesis. Moreover, significant loss of HSC quiescence and long-term reconstituting ability are observed in the absence of miR-21. Further studies revealed that miR-21 deficiency markedly decreases the nuclear factor kappa B (NF-кB) pathway, accompanied by increased expression of PDCD4, a direct target of miR-21, in HSC. Interestingly, overexpression of PDCD4 in wild-type HSC generates similar phenotypes as those of miR-21-deficient HSC. More importantly, knockdown of PDCD4 can significantly rescue the attenuation of NF- $\kappa B$ activity, thereby improving the defects in miR-21-null HSC. On the other hand, we found that miR-21 is capable of preventing HSC from ionizing radiation-induced DNA damage via activation of the NF- $\mathrm{BB}$ pathway. Collectively, our data demonstrate that miR-21 is involved in maintaining HSC homeostasis and function, at least in part, by regulating the PDCD4-mediated NF- $\mathrm{-B}$ pathway and provide a new insight into radioprotection of HSC.

\section{Introduction}

Hematopoiesis is a well-organized developmental process in which hematopoietic stem cells (HSC) can self-renew and differentiate into all kinds of blood cells. ${ }^{1,2}$ Under steady-state conditions, most adult HSC are retained in a relatively undifferentiated and quiescent state in the bone marrow (BM) microenvironment, which is necessary for sustaining long-term hematopoietic function. ${ }^{3,4}$ In contrast, the perturbation of HSC homeostasis may result in hematopoietic failure, immunodeficiencies or hematologic malignancies., ${ }^{5,6}$ It was known that HSC homeostasis is tightly modulated by a complicated molecular network. In the past decades, many factors, including cell cycle proteins, transcription factors, surface receptors, epigenetic regulatory factors, metabolic regulators, long non-coding RNA and cytokines, have been found to be involved in the control of HSC homeostasis. $1,3,8,9$ However, the underlying molecular mechanism is still not completely clear.

MicroRNA (miRNA) are small non-coding RNA that participate in a wide range of biological processes by negatively controlling the expression of their target genes through post-transcriptional regulation. ${ }^{10,11}$ Previous studies have shown that miRNA have distinct expression patterns in the hematopoietic system, and specific miRNA can affect the development of different blood-cell lineages. ${ }^{12,13}$ In recent years, several miRNA, such as miR-22, miR-29a, miR-125a, miR-126, and the miR$132 / 122$ cluster, ${ }^{7,1417}$ have been shown to play important roles in HSC biology.

miR-21, a well-known short RNA, has multiple physiological functions in mam- 
mals. It was found that miR-21 is involved in Gfi1-mediated modulation of myelopoiesis and regulates macrophage polarization and anti-inflammatory effects. ${ }^{18-}$ ${ }^{20}$ Besides, mice lacking miR-21 have reduced eosinophil levels in the peripheral blood (PB) and impaired eosinophil colony-forming capacity in the BM. ${ }^{21}$ Furthermore, another study revealed that miR-21 mediates hematopoietic suppression in myelodysplastic syndromes by targeting smad7, which is a negative regulator of the TGF- $\beta /$ smad pathway. ${ }^{22}$ In particular, recent studies have defined miR21 as an onco-miRNA, which is frequently upregulated in many kinds of tumors, including hematologic malignancies. ${ }^{17,23}$ Taken together, these findings demonstrate that miR-21 plays significant roles in the hematopoietic system. On the other hand, miR-21 was reported to be implicated in the biology of several types of stem cells, including mesenchymal stem cells, cancer stem cells and embryonic stem cells..$^{1724,25}$ However, the exact role of miR-21 in HSC populations is largely unclear.

In the present study, we first found that mouse HSC express high levels of miR-21 and that targeted deletion of miR-21 leads to abnormal hematopoiesis. Furthermore, miR-21 deficiency significantly impairs the quiescence and long-term reconstituting function of HSC. We demonstrated that miR-21 is involved in the maintenance of HSC homeostasis and function through modulation of the nuclear factor kappa B (NF-кB) pathway by regulating programmed cell death 4 (PDCD4). Of note, miR-21 is also able to mitigate radiation-induced DNA damage in HSC. Collectively, these data indicate a key role for miR-21 in HSC biology and therefore broaden our knowledge of the physiological functions of miR-21.

\section{Methods}

\section{Animals}

Normal, wild-type (WT) C57BL/6 mice were purchased from the Institute of Zoology (Chinese Academy of Sciences, Beijing, China). miR-21 $1^{\text {fox } /+}\left(\mathrm{miR}-21^{\mathrm{B} / \mathrm{+}}\right)$ mice and Mx1-Cre mice were obtained from Shanghai Model Organisms Center (China). miR21//1; $M x 1-C r e ~ m i c e ~ w e r e ~ g e n e r a t e d ~ b y ~ c r o s s i n g ~ m i R-21^{\mathrm{A} / \mathrm{I}}$ mice with Mx1-Cre mice. Unless otherwise stated, miR-21 deletion was induced by intraperitoneally injecting 4- to 6-week old miR$21^{\mathrm{A} / \mathrm{f}} ; \mathrm{Mx} 1-\mathrm{Cre}^{+}$mice with $250 \mu \mathrm{g}$ of polyinosinic:polycytidylic acid (pIpC) (Sigma, St. Louis, MO, USA) every other day for a total of seven doses. Four weeks after pIpC treatment, these mice were used in subsequent experiments. Identically treated miR$21^{\mathrm{A} / \mathrm{A}} ; \mathrm{Mx} 1-\mathrm{Cre}$ littermates served as controls. Congenic C57Bl/6 SJL CD $45.1^{+}$mice were kindly provided by Prof. Jinyong Wang (Guangzhou Institutes of Biomedicine and Health, Chinese Academy of Science, Guangzhou, China). All animal experiments were approved by the Animal Care Committee of The Third Military Medical University (Chongqing, China).

\section{Flow cytometry}

Single-cell suspensions of BM, spleen and $\mathrm{PB}$ were prepared as we described previously, ${ }^{8,26}$ Detailed information about the antibodies used for flow cytometric analyses is provided in Online Supplementary Table S1. The cell cycle, apoptosis, in vivo 5-bromodeoxyuridine incorporation and intracellular protein staining were analyzed as we reported elsewhere. ${ }^{8}$ Samples were detected using a FACSverse or FACSCanto flow cytometer (BD Biosciences, San Jose, CA, USA) and data were analyzed using FlowJo10.0 software (TreeStar, San Carlos, CA, USA). Cell sort- ing was performed using a FACSAriaII or FACSAriaIII sorter (BD Biosciences).

\section{Lentiviral transduction}

The recombinant lentivirus carrying the $P D C D 4$ gene or specific short hairpin RNA (shRNA) against PDCD4, as well as the corresponding negative controls, were obtained from Hanbio Co. Ltd. (Shanghai, China). The lentivirus was then transduced into HSC as we described previously. ${ }^{8}$ Subsequently, transduced LinSCA1 $1^{+}$c-KIT ${ }^{+}(\mathrm{LSK})$ cells $\left(6 \times 10^{3}\right)$ were purified with GFP expression through flow cytometry, and transplanted into 10.0 Gy-irradiated CD 45.1+ WT recipients along with $5 \times 10^{5} \mathrm{CD} 45.1^{+}$competitor BM cells. The sequence of sh-PDCD4 is as follows: 5'-GAGCTTGTATATGAAGCCATTGTAA-3'.

\section{Microarray analysis}

Total RNA was isolated from freshly sorted miR-21 ${ }^{\mathrm{Alt}}$ or miR$21^{\Delta \Delta}$ LSK cells. After that, samples were hybridized on Mouse Clariom D arrays (Affymetrix, Santa Clara, CA, USA) in triplicate using the procedures described in the User Manuals. Raw data were then normalized using the robust multi-array average. Genes with a fold change in expression $>1.4$ and a $P$ value $<0.05$ were defined as differentially expressed and are listed in Online Supplementary Table S3. Gene Ontology (GO) and Kyoto Encyclopedia of Genes and Genomes (KEGG) pathway enrichment were used to analyze the microarray data. The data have been deposited in the Gene Expression Omnibus database (accession number GSE131603).

\section{Statistical analysis}

All results were analyzed using GraphPad Prism 6.0 software (La Jolla, CA, USA). Differences in data between two groups and multiple groups were analyzed by a two-tailed Student $t$-test and one-way analysis of variance, respectively. The survival rates were compared by a log-rank nonparametric test and displayed as Kaplan-Meier survival curves. Unless otherwise stated, data were obtained from at least three independent experiments. $P$ values $<0.05$ were defined as statistically significant.

\section{Results}

\section{miR-21 is enriched in hematopoietic stem cells and its conditional ablation skews hematopoietic differentiation}

Previous miRNA expression profiling studies have shown that miR-21 is highly expressed in mouse BM..$^{12,27,28}$ To evaluate the role of $\mathrm{miR}-21$ in hematopoiesis, we first measured the expression of miR-21 in murine hematopoietic stem and progenitor cells (HSPC). It was found that the expression of miR-21 was relatively enriched in HSC compared with that in committed hematopoietic progenitors (Figure 1A), which hints that miR-21 may play a potential role in HSC biology. We then generated a conditional knockout mouse model (miR-21 $1^{\text {fl/til }} \mathrm{Mx} 1-\mathrm{Cre}$ ) by crossing miR-2 $1^{\mathrm{f} / \mathrm{l} l}$ mice with $\mathrm{Mx} 1-$ Cre mice (Figure $1 \mathrm{~B}$ ). The deletion of miR-21 was induced in the hematopoietic compartment by seven injections of $\mathrm{pIpC}$ (hereafter, miR$21^{\mathrm{E} / \mathrm{fl}} ; \mathrm{Mx} 1-\mathrm{Cre}$ and $\mathrm{miR}-21^{\mathrm{f} / \mathrm{fl}} ; \mathrm{Mx} 1-\mathrm{Cre}^{+}$mice are referred to as $\mathrm{miR}-21^{\mathrm{f} / \mathrm{l}}$ and miR-21 $1^{\Delta / \Delta}$ mice, respectively), which was confirmed by genomic polymerase chain reaction (PCR) and quantitative real-time PCR (qRT-PCR) analysis (Figure 1C, D; Online Supplementary Figure S1D). However, we did not find significant differences in total cell number in the BM and spleen, or in the counts of 
white blood cells, red blood cells and platelets in the PB, after miR-21 knockout (Online Supplementary Figure S1E, F). Interestingly, miR-21 $1^{\Delta \Delta}$ mice exhibited a significant increase in the percentage of myeloid cells but a marked decrease in the percentage of $\mathrm{B}$ cells in the $\mathrm{BM}$ and $\mathrm{PB}$ (Figure 1E; Online Supplementary Figure S1G). Consistently, we found that the percentage of granulocyte-monocyte progenitors (GMP) was obviously enhanced, while the frequencies of common myeloid progenitors (CMP), megakaryocyte-erythroid progenitors (MEP) and common lymphoid progenitors (CLP) were reduced in the BM after miR-21 deletion (Figure 1F, G). These findings suggest that miR-21 is crucial for steady-state hematopoiesis.

Targeted deletion of miR-21 generates an aberrant hematopoietic stem cell pool

To determine how miR-21 affects hematopoiesis, we next analyzed the phenotypes of HSC from miR-21 $1^{\Delta \Delta}$ mice
A

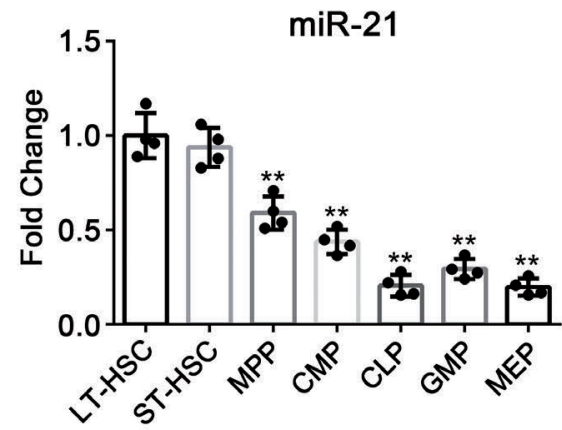

C

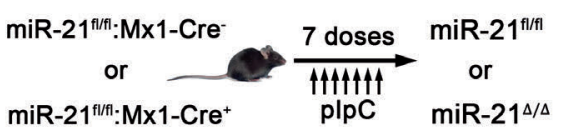

D

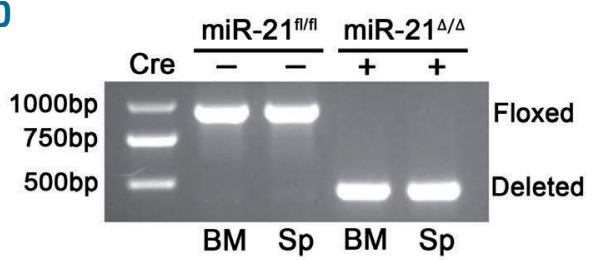

F

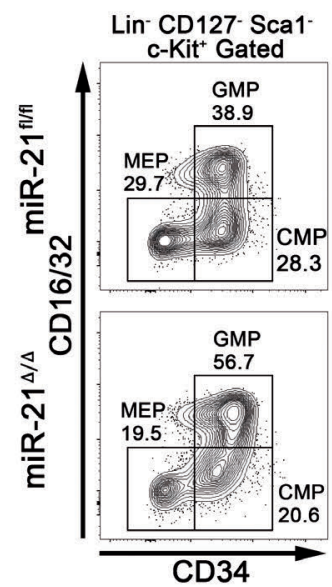

B

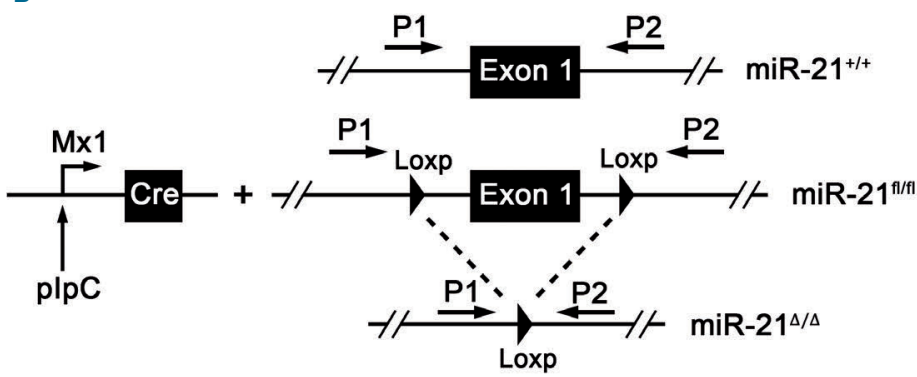

E
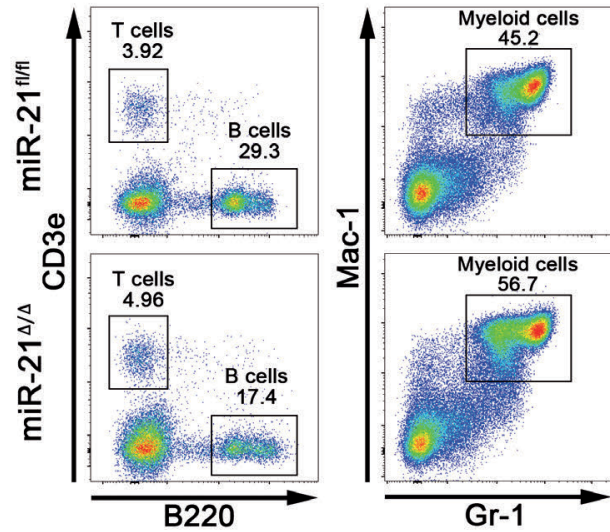

G

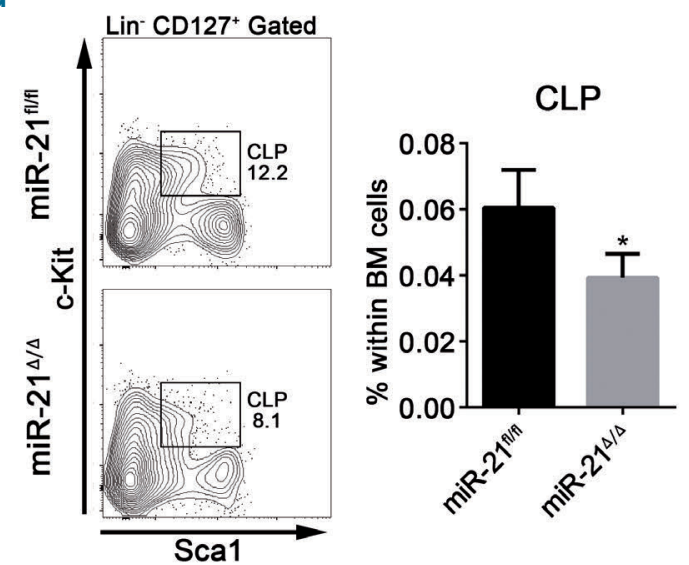

Figure 1. miR-21 is enriched in hematopoietic stem cells and its conditional ablation skews hematopoietic differentiation. (A) Quantitative real-time polymerase chain reaction (PCR) analysis of miR-21 expression in long-term hematopoietic stem cells (LT-HSC), short-term hematopoietic stem cells (ST-HSC), multipotent progenitors (MPP), common myeloid progenitors (CMP), common lymphoid progenitors (CLP), granulocyte-monocyte progenitors (GMP) and megakaryocyte-erythroid progenitors (MEP) isolated from normal 8-week old wild-type mice ( $n=4$ mice). miR-21 expression was compared with that in LT-HSC. Gating strategies are provided in Online Supplementary Figure S1A, B. (B) The strategy for the generation of the conditional miR-21 knockout mouse model. For detailed genotyping see Online Supplementary Figure S1C. (C) Schematic for plpC-inducible deletion of miR-21 in the hematopoietic system. (D) PCR-based analysis of genomic DNA from the bone

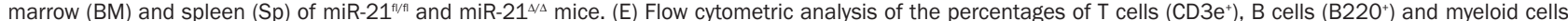

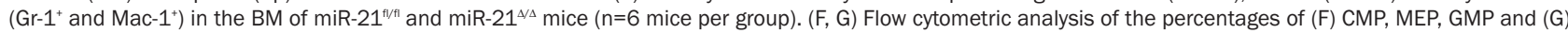

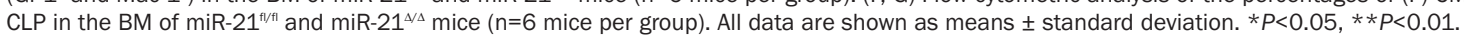


by flow cytometry. There were evident increases in the percentage and number of LSK, but not lineage-negative cells and myeloid progenitors, in the BM from miR-21 $1^{{ }^{\Delta \Delta}}$ mice compared with those in the BM of miR-21/f/l mice (Figure 2A). Further analysis revealed that the frequency of longterm HSC (LT-HSC) was increased, whereas the proportion of multipotent progenitors was decreased in the LSK compartments in the absence of miR-21 (Figure 2B). Indeed, the numbers of three LSK subpopulations, especially the LTHSC, were markedly increased in the BM after miR-21 knockout (Figure 2C). A similar result was obtained by staining LSK with another set of HSC surface markers, CD150 and CD48 (Figure 2D; Online Supplementary Figure $S 2 A)$. We also observed a dramatic increase in the percentage of LSK in the spleen, but not in the PB, when miR-21 was deleted (Figure 2E; Online Supplementary Figure S2B). These results indicate that miR-21 is responsible for sustaining the normal HSC pool.

\section{Loss of miR-21 impairs the quiescence and facilitates the proliferation of hematopoietic stem cells}

We then set out to explore the possible reasons for the accumulation of phenotypic HSC after miR-21 knockout. Actually, we found a minor but not significantly different decrease in the apoptosis of HSC upon miR-21 ablation (Online Supplementary Figure S3A). Importantly, cell cycle and in vivo 5-bromodeoxyuridine incorporation analysis revealed that miR-21 deficiency strikingly reduced the quiescence and increased the proliferation of HSC, but not of myeloid progenitors (Figure 3A, B; Online Supplementary

A
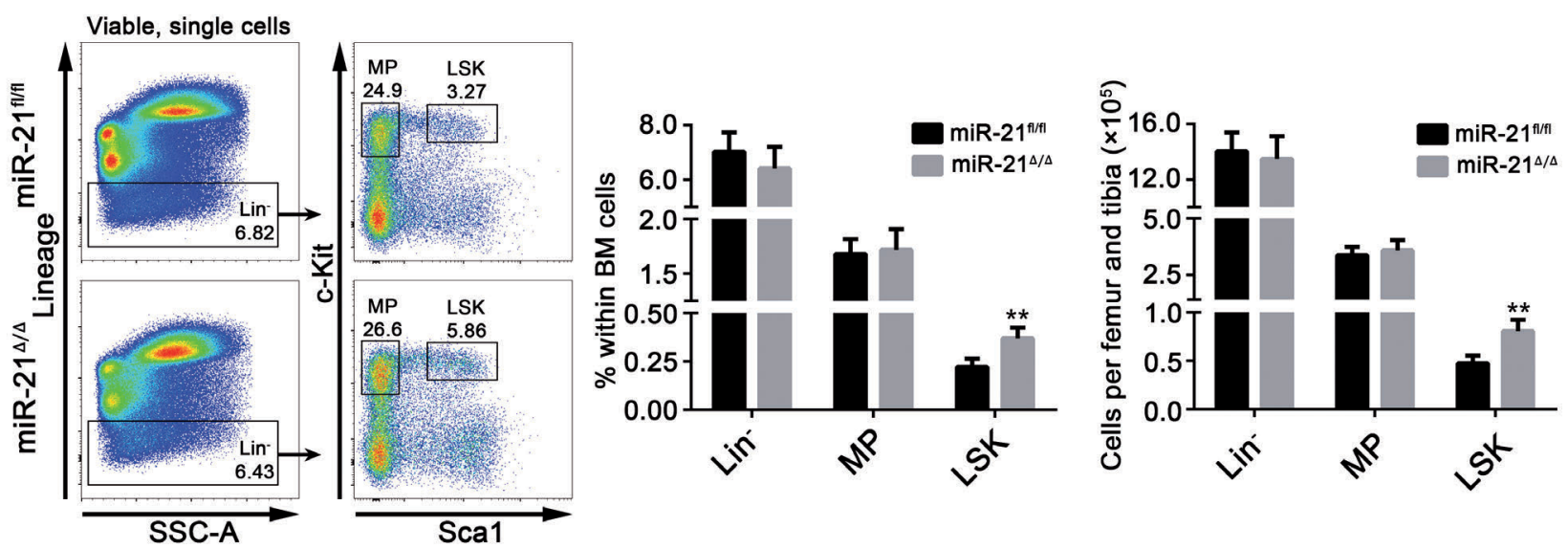

B
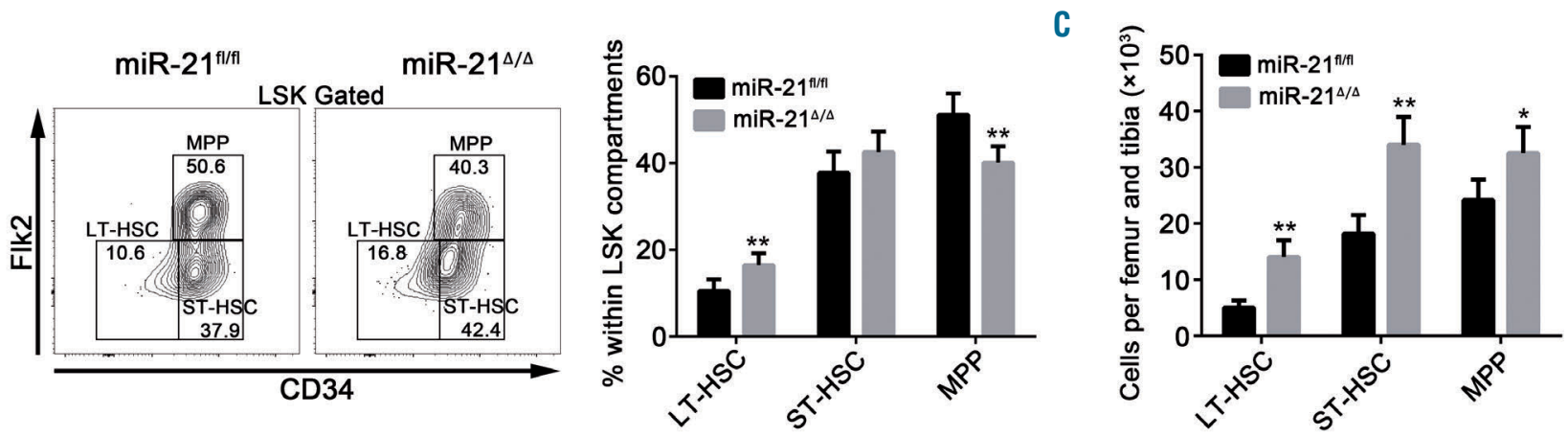

D
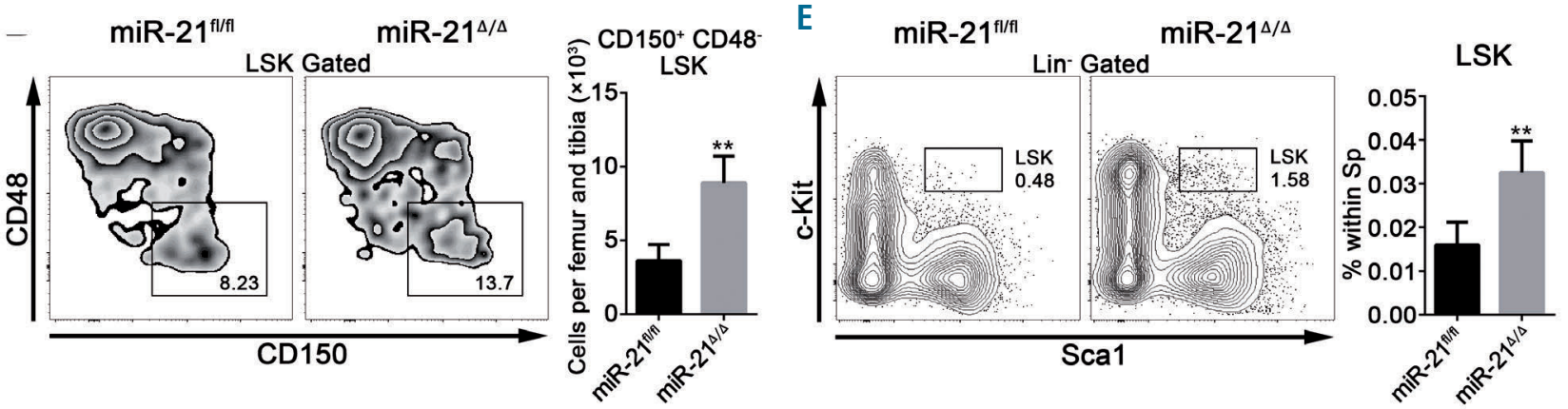

Figure 2. Targeted deletion of miR-21 generates an aberrant hematopoietic stem cell pool. (A) Flow cytometric analysis of the percentages and numbers of lineage-

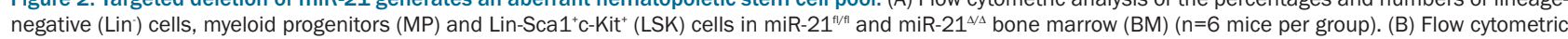
analysis of the proportions of long-term hematopoietic stem cells (LT-HSC), short-term hematopoietic stem cells (ST-HSC) and multipotent progenitors (MPP) in LSK

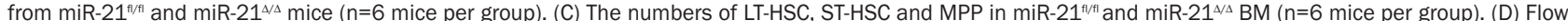
cytometric analysis of the number of CD150+ CD48 LSK in miR-21fl/fl and miR-21//s BM ( $n=6$ mice per group). (E) Flow cytometric analysis of the percentage of LSK

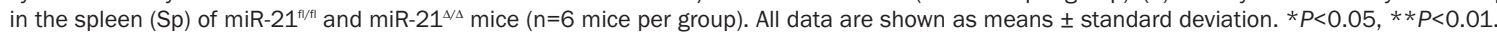


Figure $S 3 B, C)$, which is in line with the expression pattern of miR-21. Moreover, qRT-PCR data showed that cyclindependent kinase inhibitors (p21 and p27) were downregulated, whereas cell cycle-associated genes (cyclin E1, cyclin $\mathrm{E} 2$, cyclin A2 and CDK6) were upregulated in LT-HSC in the absence of miR-21 (Figure 3C).

Given that proliferating active cells are highly sensitive to genotoxic stress, ${ }^{29}$ we therefore administered miR-21 ${ }^{\text {t// }}$ and miR-21 ${ }^{\Delta \Delta}$ mice with a single dose of 5-fluorouracil. As expected, miR-21 $1^{\Delta / \Delta}$ mice displayed more severe myelosup- pression compared with that of control mice (Figure 3D, E). Likewise, the survival rate of $\mathrm{miR}-21^{\Delta \Delta}$ mice was significantly reduced after weekly 5 -fluorouracil injections (Figure $3 F)$. Taken together, these data demonstrate that miR-21 is required to maintain HSC in a quiescent state.

miR-21 deficiency intrinsically compromises hematopoietic stem cell function in long-term reconstitution

It has been well accepted that the maintenance of HSC
A

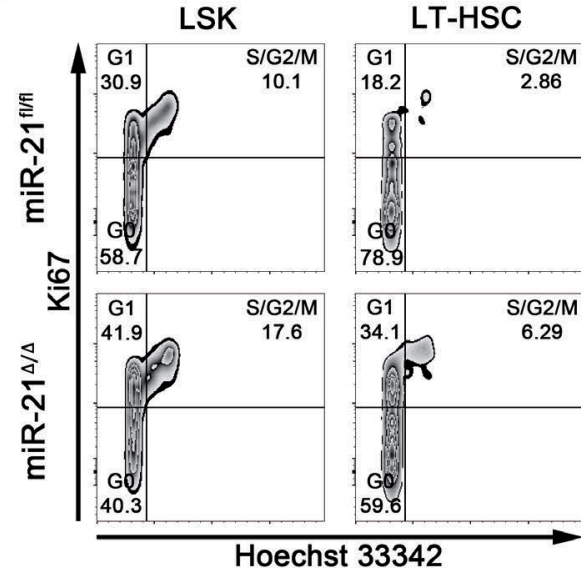

B

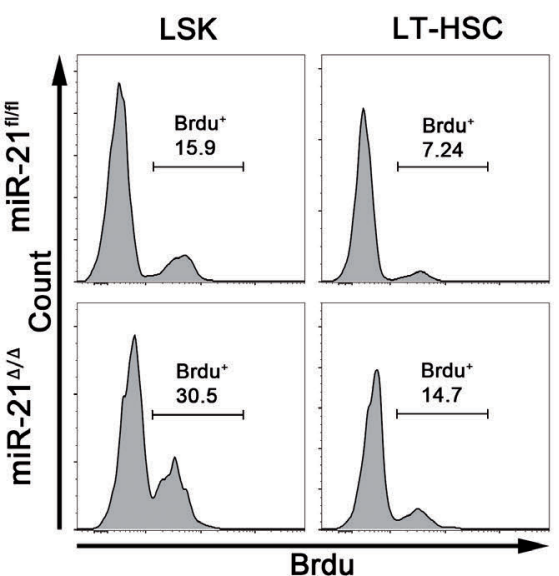

D

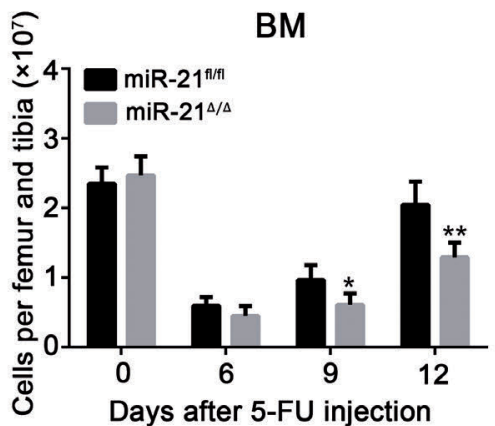

LT-HSC
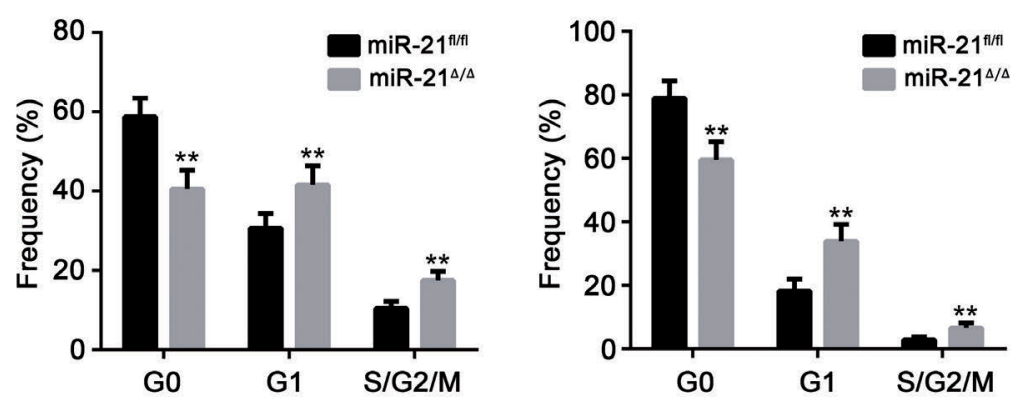

C

$\mathrm{E}$

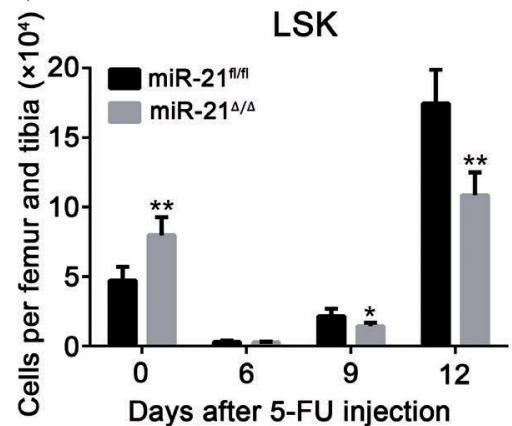

LT-HSC

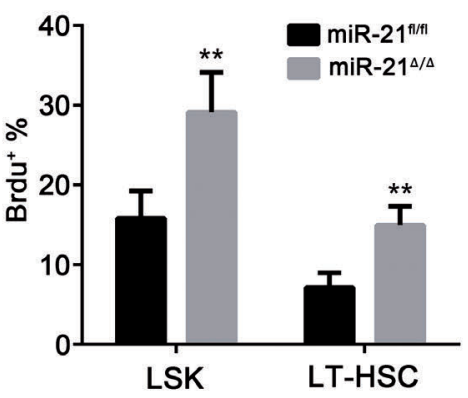

$\mathbf{F}$

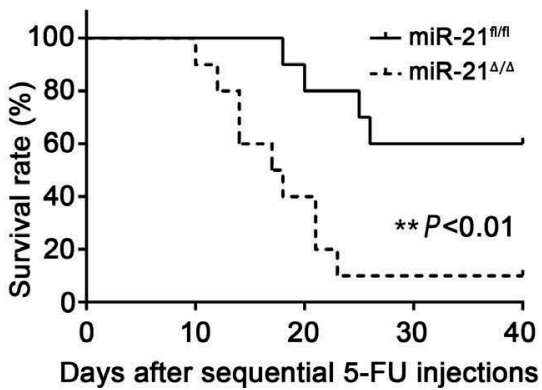

Figure 3. Loss of miR-21 impairs the quiescence and facilitates the proliferation of hematopoietic stem cells. (A) Cell cycle analysis of Lin Sca $1^{+} \mathrm{C}-\mathrm{Kit}{ }^{+}$(LSK) cells

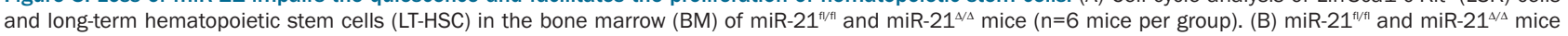
were intraperitoneally injected with 5-bromodeoxyuridine (BrdU: $1 \mathrm{mg} / 6 \mathrm{~g}$ mouse weight). Twelve hours later, BrdU incorporation into LSK and LT-HSC from these mice was analyzed by flow cytometry ( $n=6$ mice per group). (C) Quantitative real-time polymerase chain reaction analysis of the relative expression of cyclin-dependent kinase inhibitors and cell cycle-associated genes in sorted miR-21/t/f and miR-21 ${ }^{\Delta / \Delta}$ LT-HSC ( $n=3$ mice per group). (D, E) The number of BM cells (D) and LSK (E)

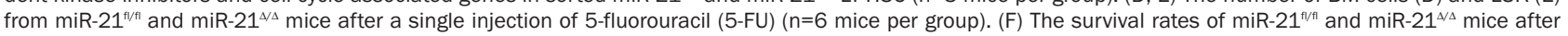
sequential 5-FU injections ( $\mathrm{n}=10$ mice per group). All data are shown as means \pm standard deviation. $* P<0.05, * * P<0.01$. 
quiescence is closely connected with these cells' long-term reconstituting capability. ${ }^{6,29}$ To study this, we first conducted non-competitive bone marrow transplantation (BMT) assays (Figure 4A). Mice transplanted with miR-21 $1^{\Delta / \Delta} \mathrm{BM}$ cells died earlier than those transplanted with miR-21 $1^{\mathrm{t} / \mathrm{I}}$ BM cells in secondary non-competitive BMT (Figure 4B, C). To further determine the function of miR-21 $1^{\Delta / \Delta}$ HSC, we next performed competitive BMT assays (Figure 4D). It was observed that miR-21 deficiency led to a reduced percentage of donor-derived cells in recipients' PB and LSK compartments after primary and secondary transplants, accompanied by myeloid-biased hematopoietic differentiation (Figure 4E-H; Online Supplementary Figure S4A, B).

To substantiate these findings, we performed another competitive BMT. BM cells from miR-21 ${ }^{\mathrm{f} / \mathrm{fl}} ; \mathrm{Mx} 1-\mathrm{Cre}$ or miR-21 ${ }^{\mathrm{t} / \mathrm{f} / \mathrm{Mx}}$ $\mathrm{M}-\mathrm{Cre}^{+}$mice which were not administered pIpC were transplanted, and at 4 weeks after transplantation, miR-21 ablation was induced by injecting pIpC into the recipients (Figure 4I). Consistently, the compromised function and biased differentiation were also observed (Figure 4J, K). Furthermore, the reciprocal BMT experiment indicates that $\mathrm{BM}$ microenvironmental changes are not enough to mediate the functional defects in miR-21 $1^{\Delta / \Delta}$ HSC (Online Supplementary Figure S4C-E). Collectively, these data confirm that miR-21 intrinsically modulates the function of HSC.

\section{Specific knockout of miR-21 dramatically decreases the NF- $\kappa$ B pathway in hematopoietic stem cells}

To gain insight into the underlying mechanisms by which miR-21 regulates HSC homeostasis and function, we conducted a microarray analysis of sorted LSK from miR-21 $1^{\mathrm{flal}}$ and miR-21 $1^{\Delta \Delta}$ mice. As shown in Figure 5A, we identified 1,050 differentially expressed genes, of which 495 genes were upregulated and 555 genes were downregulated in HSC when miR-21 was deleted. GO enrichment analysis showed that the upregulated genes were markedly enriched in nucleosome assembly, mitotic nuclear division, cell division, DNA replication-dependent nucleosome assembly, cell cycle and chromosome segregation (Figure 5B), which was in accordance with the findings that miR-21 knockout reduced the quiescence and promoted the proliferation of HSC. Besides, consistent with the myeloid bias that manifested in $\mathrm{miR}-21^{\Delta \Delta}$ mice, we noticed a robust increase in the expression of myeloid differentiation genes, such as Mpo, Fcgr3, Csf1r, Igsf6, Lybc1 and Ms4a3, ${ }^{30,31}$ in HSC after miR-21 knockout (Online Supplementary Figure S5).

Importantly, KEGG pathway analysis revealed that miR-21 ablation dramatically decreased the NF- $\mathrm{B}$ pathway in HSC (Figure 5C), which has been reported to play a critical role in maintaining hematopoietic homeostasis. ${ }^{30,32}$ In fact, a marked downregulation of NF- $\mathrm{KB}$ downstream genes was observed in miR-21 ${ }^{\Delta / \Delta}$ LT-HSC (Figure $5 \mathrm{D})$, including p21 (Figure 2C). The attenuation of NF- $\mathrm{\kappa B}$ activity was further verified by western blotting, immunofluorescence and flow cytometry (Figure 5E-G). Notably, the defects displayed in miR-21 $1^{\Delta \Delta}$ mice, including myeloid bias, accumulation of HSC in the BM and spleen, and reduced quiescence and impaired reconstituting capacity, resemble such aspects of hematopoietic p65-null mice. ${ }^{30}$ Altogether, these results suggest that miR-21 deficiency inhibits the NF- $\mathrm{KB}$ pathway in HSC, which may contribute to the defects in HSC.
The upregulation of PDCD4 is responsible for the defects in miR-21-null hematopoietic stem cells

Considering that miRNA negatively regulate gene expression at the post-transcriptional level, we then analyzed the expression of miR-21 target genes throughly. Of the miR-21 target genes that have a potential role in affecting NF- $\kappa \mathrm{B}$ activity, ${ }^{25,33-35}$ PDCD4, but not PTEN, Spry1 or Spry2, was markedly upregulated in HSC with miR-21 deficiency (Figure 6A-C; Online Supplementary Figure S6A). The binding between miR-21 and the PDCD4 3' untranslated region was then confirmed by luciferase reporter assay (Online Supplementary Figure S6B). Intriguingly, we observed that PDCD4 expression was abundant in HSC in mouse BM (Online Supplementary Figure S6C), which is consistent with the specific role of miR-21 in HSC.

We next sought to determine whether the upregulation of PDCD4 in HSC contributes to the decreased NF- $\mathrm{KB}$ activity, and defective phenotype and function observed in miR-21 $1^{\Delta / \Delta}$ mice. For this purpose, we transduced normal HSC from WT mice with a lentivirus expressing PDCD4 and found that NF- $\kappa B$ activity was significantly inhibited after overexpression of PDCD4 (Figure 6D). As a consequence, ectopic expression of PDCD4 reduced the quiescence and long-term repopulating potential of HSC, and also biased differentiation (Figure 6E, F; Online Supplementary Figure $S 6 D, E$ ). To further verify this notion, miR-2 $1^{{ }^{\Delta \Delta}}$ HSC were transduced with a lentivirus carrying shRNA against PDCD4 (Online Supplementary Figure S6F). As expected, knockdown of PDCD4 partly rescued the defects in miR-21-null HSC (Figure 6H, I; Online Supplementary Figure S6G-I). These data underscore a critical role of miR-21 in supporting the NF- $\mathrm{BB}$ pathway by targeting PDCD4, which is essential for the maintenance of HSC homeostasis.

\section{miR-21 protects hematopoietic stem cells from irradiation-induced damage by activating the NF-КB pathway}

Evidence has indicated a vital role of NF- $\kappa \mathrm{B}$ signaling in mitigating irradiation-induced hematopoietic injury, ${ }^{36-38}$ which led us to speculate that miR-21 may affect irradiation-induced biological processes in HSC. To confirm this, miR-21 $1^{\mathrm{fl} / \mathrm{l}}$ and $\mathrm{miR}-21^{\Delta / \Delta}$ mice were simultaneously subjected to ionizing radiation. We found that the loss of miR-21 led to distinctly decreased DNA damage repair, accompanied by increased apoptosis, in HSC exposed to irradiation (Figure 7A, B; Online Supplementary Figure $S 7 A)$. Moreover, several NF-кB target genes (Ier3, Xrcc5 and Gadd456) involved in DNA damage repair were significantly decreased in HSC with miR-21 deficiency (Figure 7C). Additionally, a more serious decrease in the number of HSC and white blood cells and an increased death rate were observed in miR-21 $1^{\Delta / \Delta}$ mice after irradiation (Figure 7D, E; Online Supplementary Figure S7B). Based on the finding that NF- $\kappa \mathrm{B}$ signaling is implicated in thrombopoietin-promoted DNA damage repair in HSC, we administered thrombopoietin to the mice and observed that it did not work in miR-21 ${ }^{\Delta / \Delta}$ HSC (Online Supplementary Figure S7F, G).

Finally, we determined whether the addition of exogenous miR-21 can protect HSC from irradiation by activating the NF-кB pathway. Treatment of mice with a miR-21 agomir, which is an engineered miRNA mimic, increased NF- $\kappa \mathrm{B}$ activity in HSC by suppressing PDCD4 (Figure 
$7 \mathrm{H})$, thereby significantly alleviating irradiation-induced damage (Figure 7J; Online Supplementary Figure S7C). These effects were abrogated by a specific NF- $\mathrm{BB}$ inhibitor, ONZ ${ }^{38}$ (Figure 7J; Online Supplementary Figure $S 7 C$ ). In conclusion, our findings demonstrate that miR21 can also protect HSC from radiation damage.

\section{Discussion}

The maintenance of HSC homeostasis contributes to the continuous supplementation of blood cells throughout the lifetime. ${ }^{1,6}$ In the past few decades, studies aimed at researching hematopoiesis have revealed many factors
A

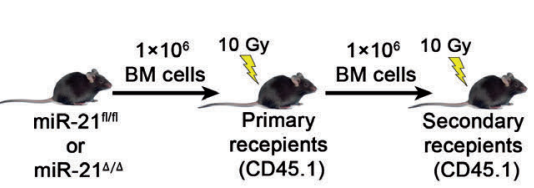

D

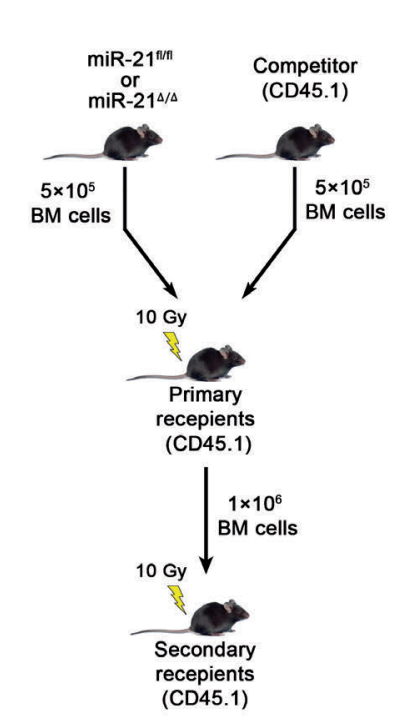

I

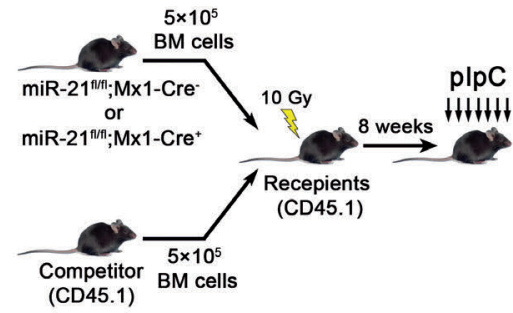

E

G

J
B
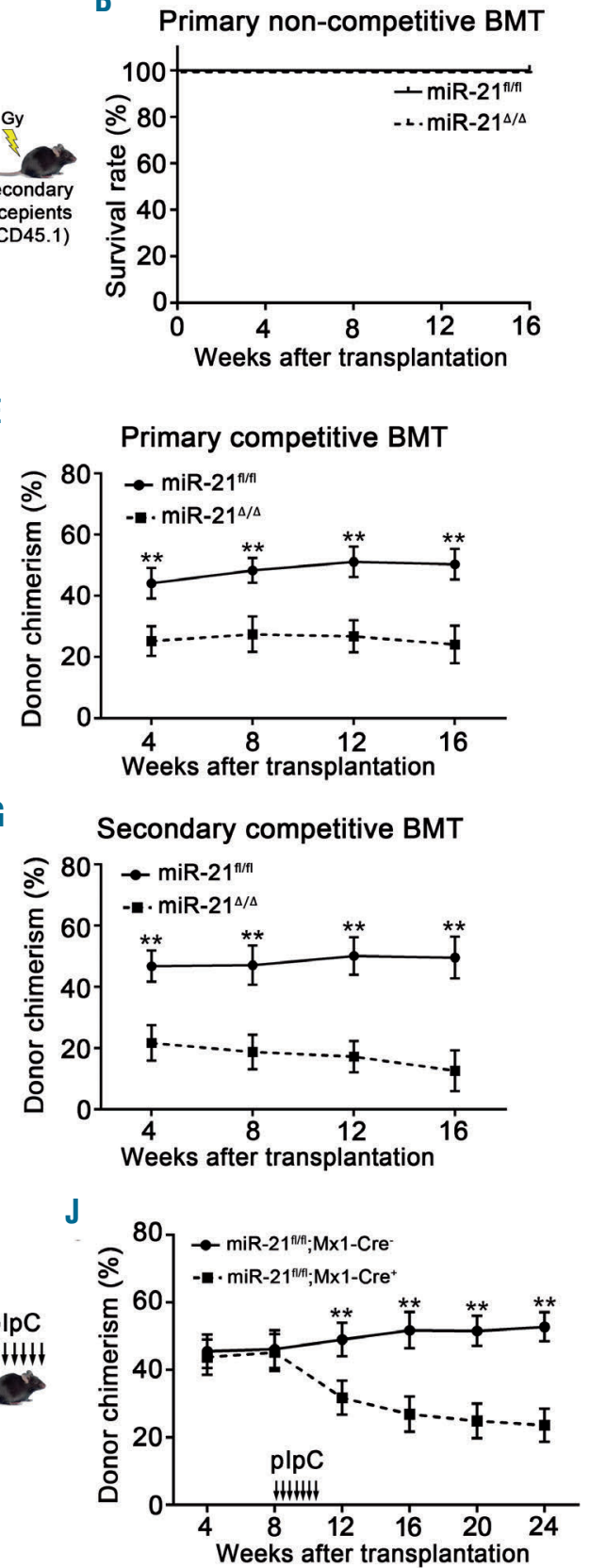

C

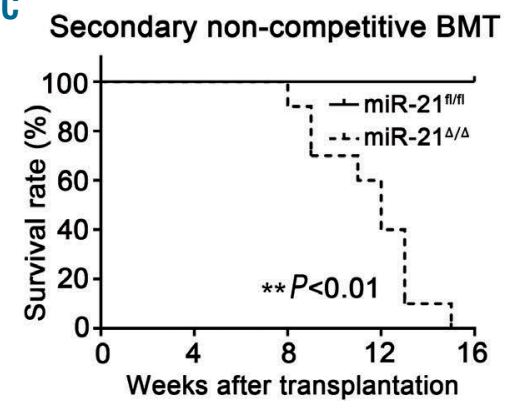

F

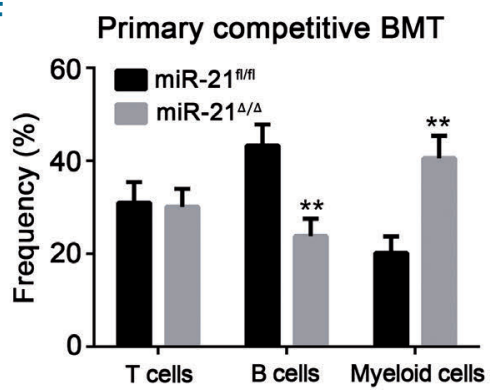

H

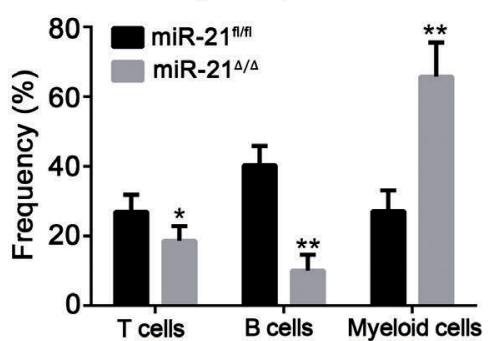

K

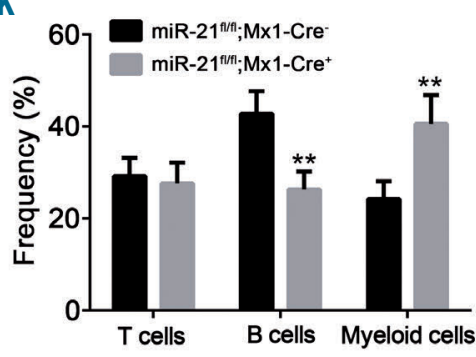

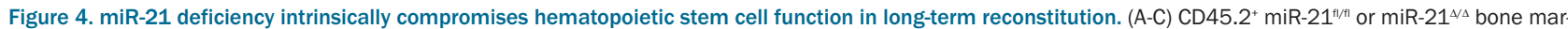
row (BM) cells were transplanted into 10.0 Gy-irradiated CD45.1 recipients. Sixteen weeks later, BM cells harvested from primary recipients were transplanted into 10.0 Gy-irradiated secondary CD45.1 recipients. The survival rates of these recipient mice after primary (B) and secondary (C) non-competitive bone marrow trans-

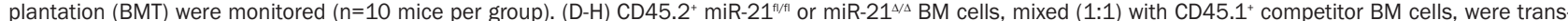
planted into 10.0 Gy-irradiated CD45.1 ${ }^{+}$recipients. Sixteen weeks later, BM cells harvested from primary recipients were transplanted into 10.0 Gy-irradiated secondary CD45.1 recipients. At the indicated times, CD45.2+ donor chimerism and lineage distribution in recipients' peripheral blood (PB) after primary (E, F) and secondary $(\mathrm{G}, \mathrm{H})$ competitive BMT were analyzed by flow cytometry ( $\mathrm{n}=8$ mice per group). The data shown on lineage distribution were obtained at 16 weeks after primary

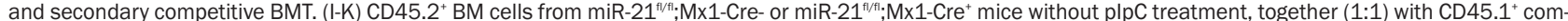
petitor BM cells, were transplanted into 10 Gy-irradiated CD45.1 WT recipients. Eight weeks after transplantation, miR-21 deletion was induced by injecting the recipients with plpC. At the indicated times, the donor chimerism $(\mathrm{J})$ and lineage distribution $(\mathrm{K})$ in recipients' PB after transplantation were analyzed by flow cytometry. The data shown on lineage distribution were obtained at 24 weeks after transplantation. A diagram is shown in the left panel. All data are shown as means \pm standard deviation. $* P<0.05, * * P<0.01$. 
capable of controlling the homeostasis of HSC. Recently, growing evidence has suggested that miRNA play prominent roles in hematopoietic cells, including HSC. ${ }^{17,27}$ In this work, we showed for the first time that miR-21 is required to maintain HSC homeostasis and function by sustaining the NF- $\kappa B$ pathway.

As a multifaceted, non-coding RNA, miR-21 is present in multiple tissues and implicated in various physiological and pathological processes. ${ }^{39}$ Here, we observed that miR21 is relatively enriched in HSC in adult mouse BM, implying that this microRNA may be involved in HSC biology. As a consequence, we found that specific knockout of miR-21 in the hematopoietic system causes an abnormal expansion of the HSC pool in the BM and spleen. Besides, mice with conditional deletion of miR-21 display a distinctly myeloid-skewed differentiation, along with a decrease of $\mathrm{B}$ cells. The reason might be that miR-
21 deficiency affects the differentiation of HSPC, a concept with is consistent with the findings of a previous study. ${ }^{18}$ In addition, a previous study showed that miR 21 can inhibit the apoptosis of diffuse large B cell lymphoma cells through upregulating BCL-2. ${ }^{40}$ Thus, miR-21 may also play a direct role in regulating B-cell survival. Taken together, our results reveal that miR-21 is essential to maintain normal hematopoiesis in mice.

Recently, miR-21 has been increasingly regarded as an oncogene due to its function in promoting tumor cell proliferation and inhibiting apoptosis. ${ }^{10}$ In the present study, we found that miR-21 deficiency leads to a marked impairment in HSC quiescence, accompanied by increased proliferation, which is very different from the functions of miR-21 in other types of cells. Additionally, deletion of miR-21 in HSC had no effect on cell apoptosis at a steady state. These results suggest that miR-21 regu-
A

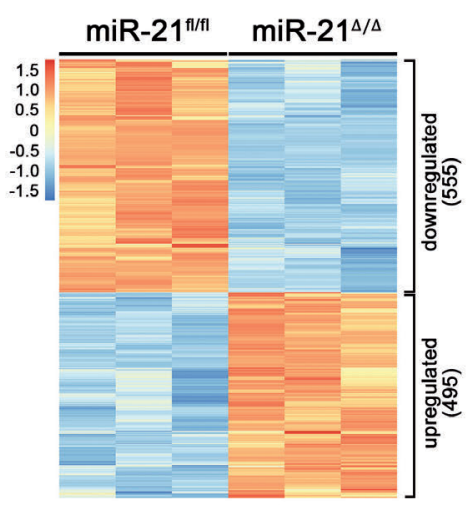

B

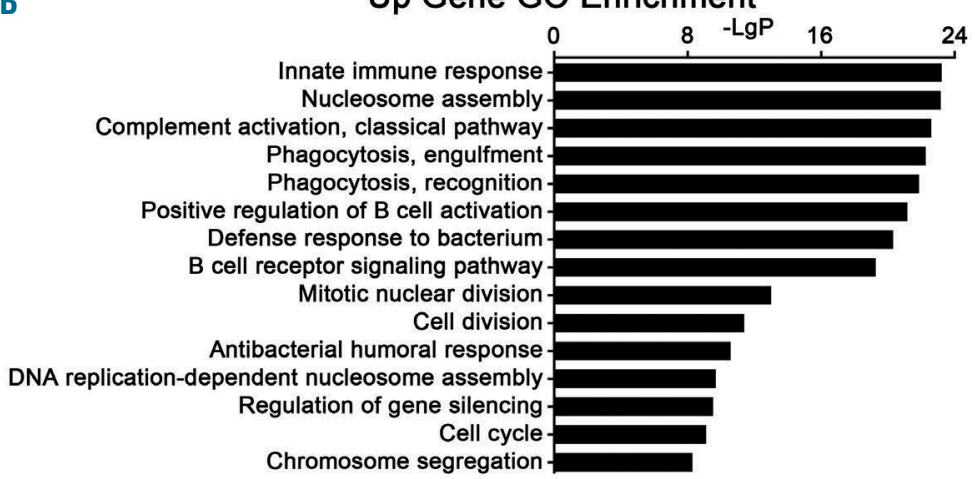

C

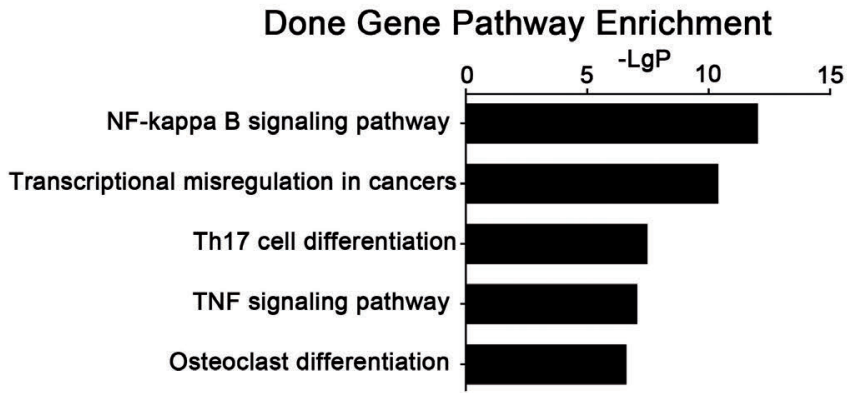

E

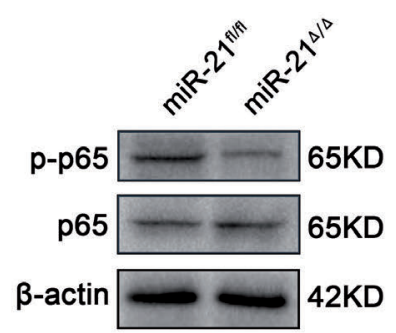

$\mathrm{F}$

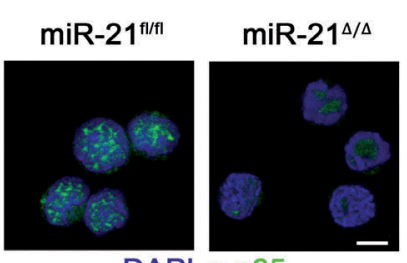

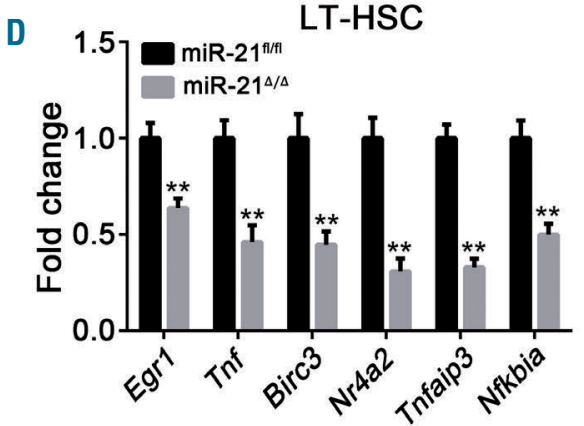

G

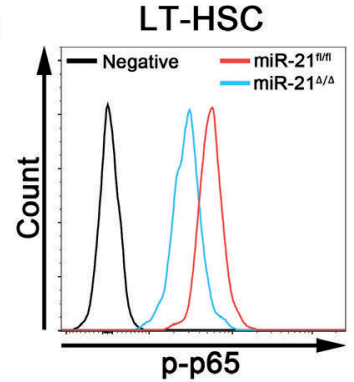

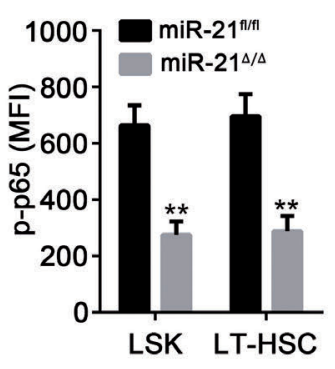

Figure 5. Specific knockout of miR-21 dramatically inhibits the NF-KB pathway in hematopoietic stem cells. (A-C) Lin-Sca1 ${ }^{+} \mathrm{C}-\mathrm{Kit}^{+}$(LSK) cells sorted from miR-21 ${ }^{\Delta /}$

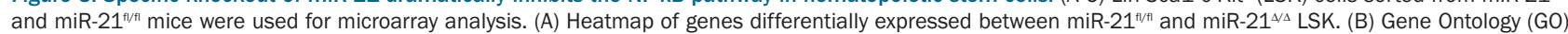
analysis of genes upregulated in LSK after deletion of miR-21. The data shown are the top 15 enriched GO terms. (C) KEGG pathway analysis of downregulated genes in miR-21 $1^{\Delta / \Delta}$ LSK relative to miR-21/ifil LSK. The top five enriched pathways are shown. The microarray data were gathered from one experiment with three biological replicates. (D) Quantitative real-time polymerase chain reaction analysis of the relative expression of NF-kB target genes (including Egr1, Tnf, Birc3, Nr4a2, Tnfaip3

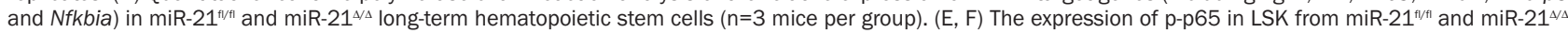
bone marrow ( $n=5$ mice per group), determined by western blotting $(E)$ and immunofluorescence (F), respectively. DAPI staining indicates the nucleus of cells. Scale bar represents $5 \mu \mathrm{m}$. (G) Flow cytometric analysis of the expression of p-p65 in LSK and LT-HSC from miR-21 ${ }^{\mathrm{t} / \mathrm{fl}}$ and miR-21 ${ }^{\Delta / \Delta}$ bone marrow ( $\mathrm{n}=5 \mathrm{mice}$ per group). MFI: mean fluorescence intensity. All data are shown as means \pm standard deviation. $* * P<0.01$. 
lates the cell cycle and apoptosis, most likely depending on the cellular contexts. It was well recognized that the loss of HSC quiescence can bring about a transient augmentation of phenotypic HSC but eventually compromises their function. ${ }^{6}$ These results could explain our observation that miR-21 $1^{\Delta \Delta}$ HSC have a diminished long-term reconstituting capacity. Notably, the reciprocal transplantation assay validated that the defects manifested in miR21-deficient HSC are cell-intrinsic. In addition, we observed a myeloid bias in recipients transplanted with miR-21 $1^{\Delta \Delta}$ BM cells, which is consistent with the changes in non-transplanted miR-21 $1^{\Delta / \Delta}$ mice.

In an effort to characterize the molecular mechanisms by which miR-21 regulates HSC homeostasis, we performed a microarray analysis. Notably, we observed a marked downregulation of the NF- $\mathrm{KB}$ pathway when
miR-21 was deleted. It is well established that the NF- $\kappa B$ transcription factor family plays a key role in various physiological processes, including cell proliferation, apoptosis, inflammation and immune responses. ${ }^{41}$ Current studies using mouse genetic models have indicated that, although aberrant activation of NF- $\mathrm{KB}$ is not beneficial for hematopoiesis, basal NF- $\kappa \mathrm{B}$ signaling is indispensable for HSC homeostasis. ${ }^{42}$ Interestingly, miR-21 ${ }^{\Delta / \Delta}$ HSC showed similar phenotypes to those of p65-null HSC. ${ }^{30}$ However, it is unknown whether miR-21 regulates HSC homeostasis and function via the NF- $\mathrm{KB}$ pathway. Further investigations revealed that a previously recognized target of miR21 , the tumor suppressor PDCD4, ${ }^{33,43}$ was obviously upregulated in HSC with miR-21 deficiency. However, there is controversy about the function of PDCD4 in reg-

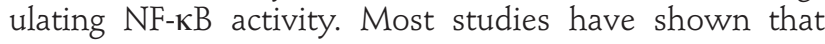

A

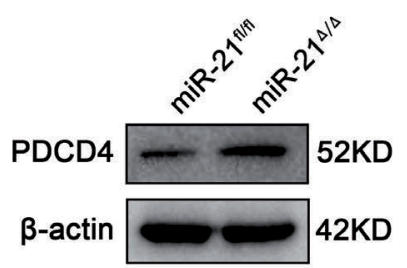

D

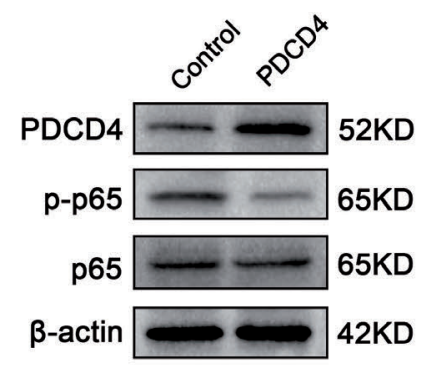

G

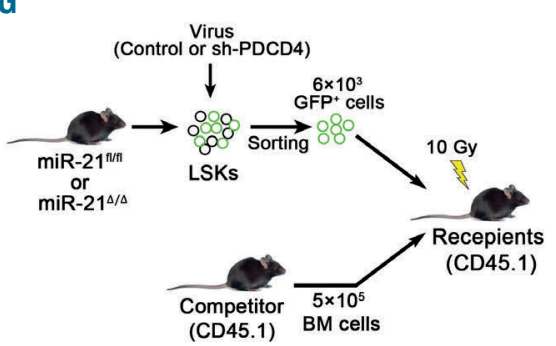

B

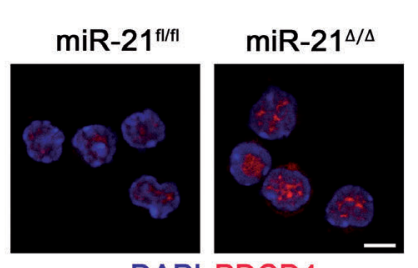

DAPI PDCD4
C

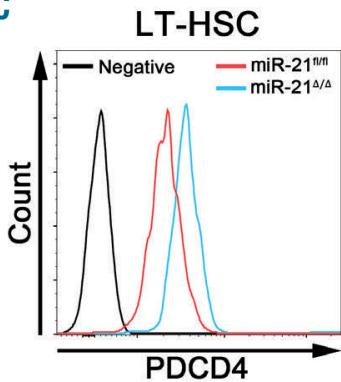

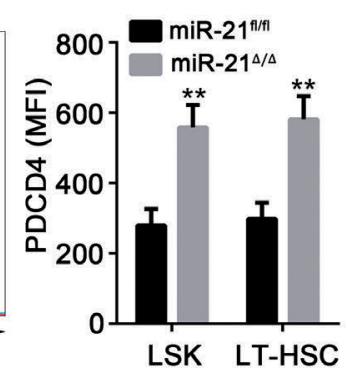

LT-HSC
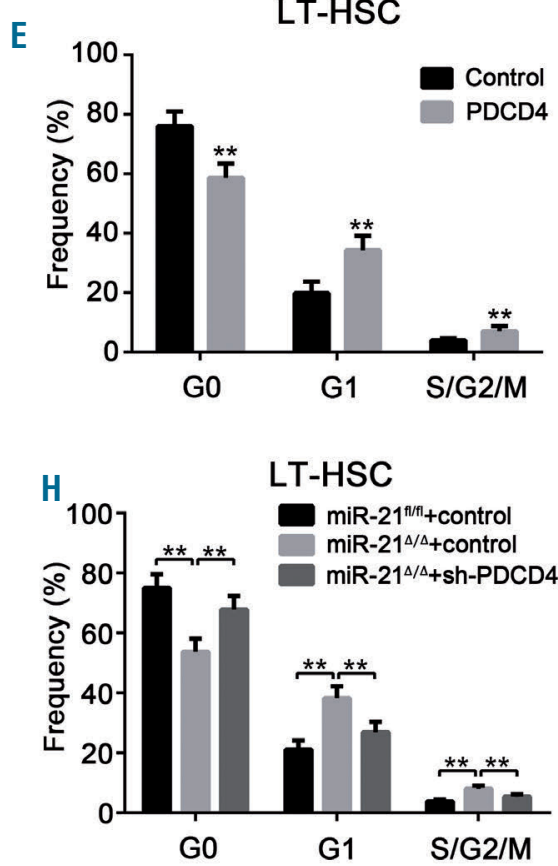
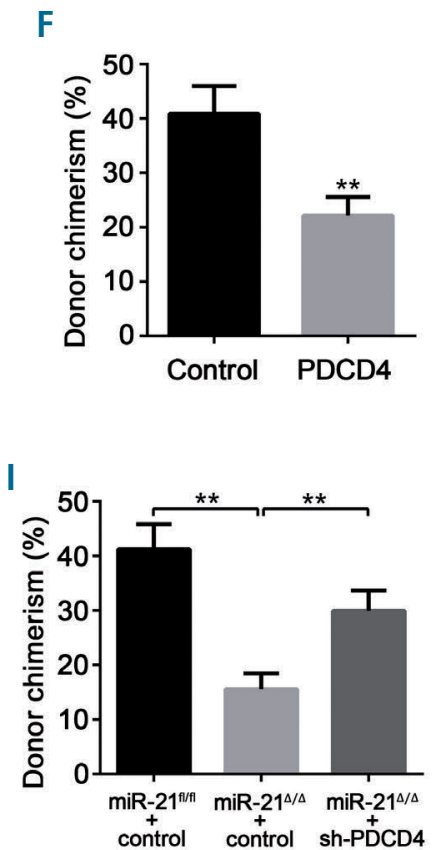

Figure 6. The upregulation of PDCD4 is responsible for the defects in miR-21-null hematopoietic stem cells. (A, B) The expression of PDCD4 in Lin Sca1 ${ }^{+} \mathrm{C}-\mathrm{Kit}^{+}$(LSK) cells from miR-21 $1^{\mathrm{f} / \mathrm{fl}}$ and miR-21 ${ }^{\mathrm{s} / \Delta}$ bone marrow (BM) ( $\mathrm{n}=5$ mice per group), determined by western blotting (A) and immunofluorescence (B), respectively. DAPI staining indicates the nucleus of cells. Scale bar represents $5 \mu \mathrm{m}$. (C) Flow cytometric analysis of the expression of PDCD4 in LSK and long-term hematopoietic stem cells (LT-HSC) from miR-21 $1^{\mathrm{H} / 1 /}$ and miR-21 ${ }^{\Delta / \Delta} \mathrm{BM}$ ( $\mathrm{n}=5$ mice per group). MFI: mean fluorescence intensity. (D) Western blotting analysis of the expression of PDCD4, p-p65 and p65 in LSK transduced with control or PDCD4 ( $n=5$ mice per group). (E, F) Normal LSK from CD45.2 ${ }^{+}$wild-type mice were transduced with the lentivirus carrying control or PDCD4, then transduced cells $\left(6 \times 10^{3}\right)$, mixed with CD $45.1^{+}$competitor BM cells $\left(5 \times 10^{5}\right)$, were transplantated into 10.0 Gy-irradiated $\mathrm{CD} 45.1^{+}$recipients. At 12 weeks after transplantation, the cell cycle of CD45.2+ donor-derived LT-HSCs in recipients' BM (E), and the CD45.2+ donor chimerism in recipients' peripheral

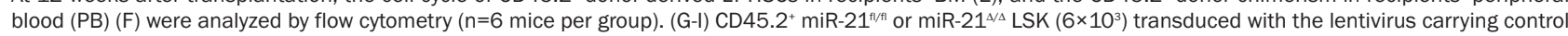
or shRNA against PDCD4 (sh-PDCD4), mixed with CD45.1 competitor BM cells $\left(5 \times 10^{5}\right)$, were transplanted into 10.0 Gy-irradiated CD45.1 recipients. At 12 weeks after transplantation, the cell cycle of CD45.2 $2^{+}$donor-derived LT-HSC in recipients' BM (H), and the CD45.2 ${ }^{+}$donor chimerism in recipients' PB (I) were analyzed by flow cytometry ( $\mathrm{n}=6$ mice per group). All data are shown as means \pm standard deviation. ${ }^{*} P<0.01$. 
PDCD4 is involved in promoting NF- $\kappa \mathrm{B}$ activation. ${ }^{44,45}$ However, in our study, we found that overexpression of PDCD4 in WT HSC by lentiviral transfection inhibited NF-кB activity, while knockdown of PDCD4 expression significantly improved the defects in miR-21-null HSC. These results elucidate that miR-21 sustains the NF- $\kappa B$ pathway, at least partly, by directly targeting PDCD4, which is consistent with the results of a previous study. ${ }^{33}$ However, whether miR-21 can regulate other target genes still needs further research. In addition, there is an opposing point of view that miR-21 inhibits NF- $\kappa B$ activity, ${ }^{45}$ which may involve miR-21 regulation of physiological functions in a cellular context-dependent manner.
Ionizing radiation-caused hematopoietic cell death is primarily attributed to the generation of double-strand breaks, which are the most serious form of DNA damage. ${ }^{2}$ At present, the acute myelosuppression induced by irradiation can be temporarily treated using various hematopoietic growth factors that are able to accelerate the recovery of hematopoietic function by stimulating HSPC proliferation and differentiation. ${ }^{46}$ However, an effective strategy to protect DNA from irradiation-induced damage in HSC is still lacking. Previous studies have revealed that miR-21 mediates resistance to radiation in many types of tumor cells, which is a great challenge for cancer radiotherapy. ${ }^{47}$ Furthermore, it has been shown that miR-21 can be stim-
A

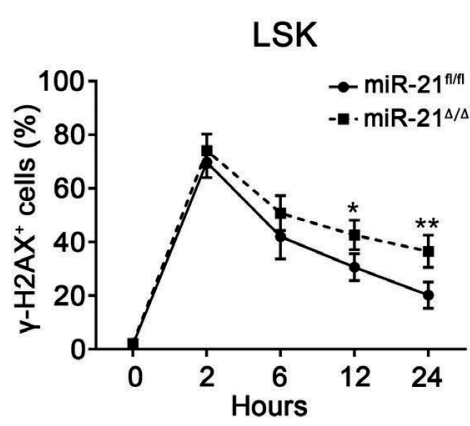

D

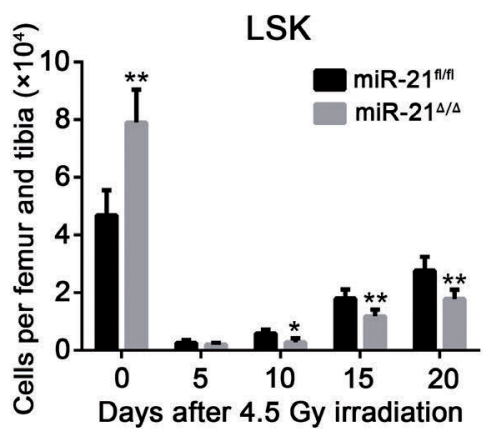

G

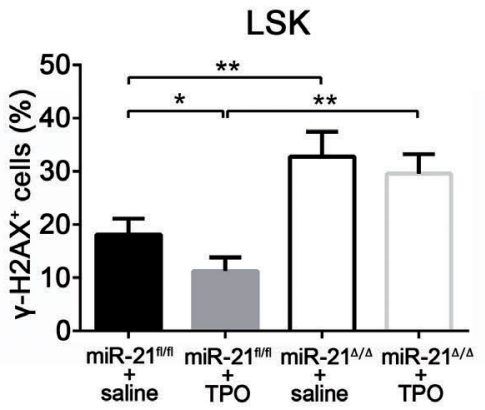

B
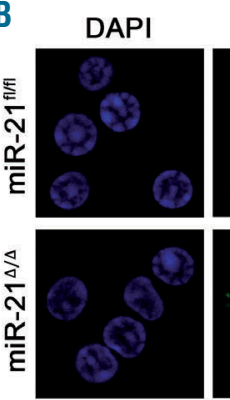

$\mathrm{E}$

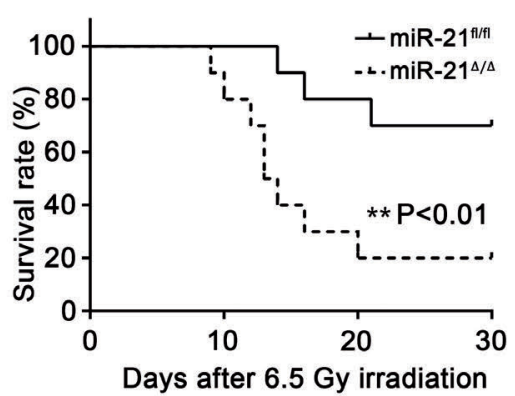

C
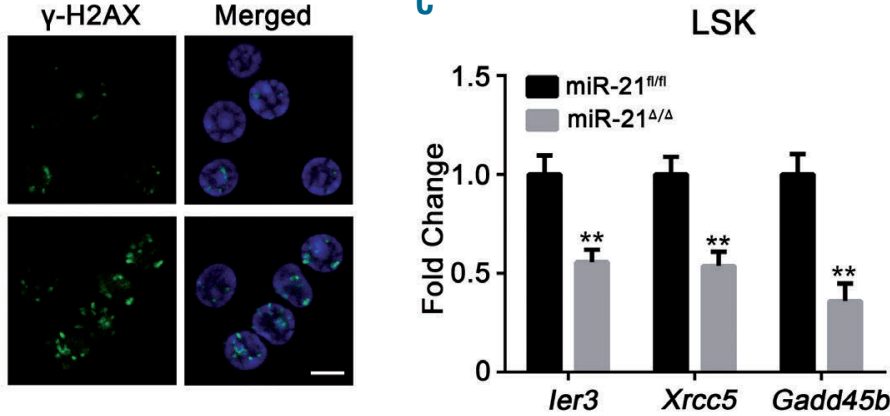

F

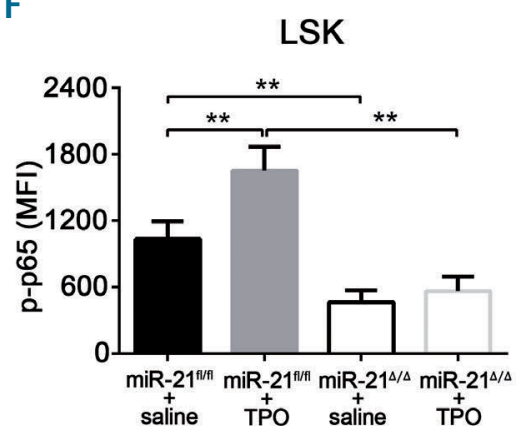

$\mathrm{H}$

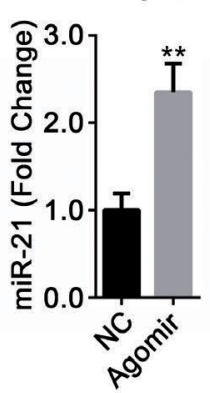

LSK

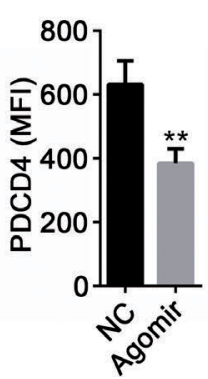

LSK

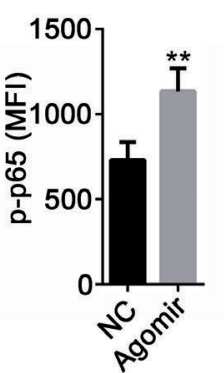

I

LSK

Figure 7. miR-21 protects hematopoietic stem cells from irradiation-induced damage by activating the NF-KB pathway. (A) Flow cytometric analysis of the percentage of $\gamma-\mathrm{H}_{2} \mathrm{AX}^{+}$cells at 0 (No irradiation) or 2, 6, 12, $24 \mathrm{~h}$ after 4.0 Gy total body irradiation (TBI) ( $\mathrm{n}=6$ mice per group). (B) Representative immunofluorescence plots showing the expression of $\gamma-\mathrm{H} 2 \mathrm{AX}$ in Lin-Sca $1^{+} \mathrm{C}-\mathrm{Kit}^{+}(\mathrm{LSK})$ cells from miR-21 $1^{\mathrm{t} / \mathrm{fl}}$ and miR-21 ${ }^{\mathrm{s} / \Delta}$ mice $24 \mathrm{~h}$ after $4.0 \mathrm{~Gy}$ TBI. DAPI staining indicates the nucleus of cells. Scale bar represents $5 \mu \mathrm{m}$. (C) Quantitative real-time polymerase chain reaction (qRT-PCR) analysis of the relative expression of NF-KB target genes (ler3, Xrcc5 and

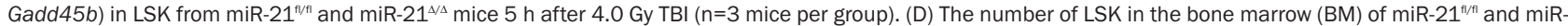
$21^{\Delta / \Delta}$ mice after 4.5 Gy TBI ( $n=6$ mice per group). (E) The survival rates of miR-21 miR-21 ${ }^{\Delta / \Delta}$ mice were treated with saline or thrombopoietin (TPO; $8 \mu \mathrm{g} / \mathrm{kg}$ ) 30 min before $4.0 \mathrm{~Gy}$ TBI. Then, the expression of p-p65 in LSK was measured 40 min after irradiation ( $n=6$ mice per group) $(F)$, and the percentage of $\gamma-H 2 A X^{+} L S K$ in these mice was measured by flow cytometry $24 \mathrm{~h}$ after irradiation ( $n=6$ mice per group) (G). (H) Normal wild-type mice were treated with three doses of miR-21 agomir or negative control (NC) on consecutive days by tail intravenous injection. Twenty-four hours after the last injection, the relative miR-21 expression in LSK from these mice was detected by qRT-PCR ( $\mathrm{n}=6$ mice per group), and the expression of PDCD4 and p-p65 in LSK from these mice was analyzed by flow cytometry ( $n=6$ mice per group). (I) Mice were treated with miR-21 agomir or NC as described before. Twentyone hours after the last injection, mice were intraperitoneally injected with one dose of QNZ or control. Three hours later, mice were subjected to 4.0 Gy TBI. The percentage of $\gamma-\mathrm{H}_{2} \mathrm{AX}{ }^{+}$cells in LSK from these mice was detected $24 \mathrm{~h}$ after irradiation by flow cytometry ( $\mathrm{n}=6$ mice per group). All data are shown as means \pm standard deviation. $* P<0.05, * * P<0.01$. 
ulated by irradiation via the EGFR/STAT3 and ATM pathways, ${ }^{48,49}$ which is line with our data (Online Supplementary Figure S7D). However, the role of miR-21 in HSC in the context of irradiation remains obscure. In the present study, we discovered that miR-21 can prevent HSC from irradiation-induced DNA damage through activating the NF-kB pathway, which is consistent with a recent finding that miR-21 promotes hematopoietic cell survival after exposure to irradiation. ${ }^{50}$ Moreover, our study revealed that miR-21 may also be involved in thrombopoietinmediated non-homologous end junction repair in HSC. Nevertheless, we do not deny that there may be other NF$\kappa \mathrm{B}$-independent mechanisms that mediate the radioprotective effects of miR-21 in HSC. ${ }^{47}$

On the other hand, some studies have reported that miR-21 is also a downstream target gene of the NF- $\mathrm{kB}$ pathway. ${ }^{44}$ Indeed, overexpression of PDCD4 or treatment with a NF- $\mathrm{kB}$ inhibitor (QNZ) significantly inhibited the expression of miR-21 in HSC from normal WT mice (data not shown). These findings led us to hypothesize that there may be a positive regulatory feedback loop that regulates HSC function.

In summary, our findings reveal a novel function of miR-21 as an important regulator of HSC homeostasis via modulation of the NF-kB pathway by targeting PDCD4, thereby extending our understanding of the biological function of miR-21. At the same time, we demonstrate that targeting miR-21 may be a promising avenue for safeguarding HSC against irradiation damage.

\section{Disclosures}

No conflicts of interest to disclose.

\section{Contributions}

$M H$ designed the study, performed experiments, analyzed data and wrote the paper; YL, ZZ and $H Z$ performed experiments and analyzed data. SC, YQ, YX, FC and YT participat$e d$ in the animal experiments and data analysis; $M C, C D$ and $M S$ contributed to the in vitro experiments; FW and YS participated in the initial experimental design and discussed the manuscript; JW and SW conceived and supervised the study, and revised the manuscript.

\section{Acknowledgments}

We thank Prof. Jinyong Wang for the gift of the CD45.1 mice, Yang Liu for technical support in flow cytometry, and Liting Wang for technical assistance in immunofluorescence microscopy. This work was supported by grants from the National Natural Science Fund of China (n. 81725019, 81930090, 81573084, 81500087) and the Scientific Research Project of PLA (AWS16J014).

\section{References}

1. Rathinam C, Matesic LE, Flavell RA. The E3 ligase Itch is a negative regulator of the homeostasis and function of hematopoietic stem cells. Nat Immunol. 2011;12(5):399407

2. Yamashita M, Nitta E, Suda T. Aspp1 preserves hematopoietic stem cell pool integrity and prevents malignant transformation. Cell Stem Cell. 2015;17(1): 23-34.

3. Suda T, Takubo K, Semenza GL. Metabolic regulation of hematopoietic stem cells in the hypoxic niche. Cell Stem Cell. 2011;9(4): 298-310.

4. Miyamoto K, Araki KY, Naka K, et al. Foxo3a is essential for maintenance of the hematopoietic stem cell pool. Cell Stem Cell. 2007;1(1):101-112.

5. Anso E, Weinberg SE, Diebold LP, et al. The mitochondrial respiratory chain is essential for haematopoietic stem cell function. Nat Cell Biol. 2017;19(6):614-625.

6. Wang H, Diao D, Shi Z, et al. SIRT6 controls hematopoietic stem cell homeostasis through epigenetic regulation of Wnt signaling. Cell Stem Cell. 2016;18(4):495-507.

7. Mehta A, Zhao JL, Sinha N, et al. The microRNA-132 and microRNA-212 cluster regulates hematopoietic stem cell maintenance and survival with age by buffering FOXO3 expression. Immunity. 2015;42(6): 1021-1032.

8. $\mathrm{Hu} \mathrm{M}$, Zeng $\mathrm{H}$, Chen S, et al. SRC-3 is involved in maintaining hematopoietic stem cell quiescence by regulation of mitochondrial metabolism in mice. Blood. 2018;132(9): 911-923.

9. Qian P, He XC, Paulson A, et al. The Dlk1$\mathrm{Gt} 2$ locus preserves LT-HSC function by inhibiting the PI3K-mTOR pathway to restrict mitochondrial metabolism. Cell Stem Cell. 2016;18(2):214-228

10. Medina PP, Nolde M, Slack FJ. OncomiR addiction in an in vivo model of microRNA21-induced pre-B-cell lymphoma. Nature.
2010;467(7311):86-90.

11. Thum T, Gross C, Fiedler J, et al. MicroRNA-21 contributes to myocardial disease by stimulating MAP kinase signalling in fibroblasts. Nature. 2008;456 (7224):980-984

12. O'Connell RM, Chaudhuri AA, Rao DS, Gibson WS, Balazs AB, Baltimore D. MicroRNAs enriched in hematopoietic stem cells differentially regulate long-term hematopoietic output. Proc Natl Acad Sci U S A. 2010;107(32):14235-14240.

13. Kotaki R, Koyama-Nasu R, Yamakawa N, Kotani A. miRNAs in normal and malignant hematopoiesis. Int J Mol Sci. 2017;18(7).

14. Song SJ, Ito K, Ala U, et al. The oncogenic microRNA miR-22 targets the TET2 tumor suppressor to promote hematopoietic stem cell self-renewal and transformation. Cell Stem Cell. 2013;13(1):87-101.

15. Hu W, Dooley J, Chung SS, et al. miR-29a maintains mouse hematopoietic stem cell self-renewal by regulating Dnmt3a. Blood. 2015;125(14):2206-2216.

16. Lechman ER, Gentner B, Ng SWK, et al. miR126 regulates distinct self-renewal outcomes in normal and malignant hematopoietic stem cells. Cancer Cell. 2016;29(4):602-606.

17. Roden C, Lu J. MicroRNAs in control of stem cells in normal and malignant hematopoiesis. Curr Stem Cell Rep. 2016;2 (3):183-196

18. Velu CS, Baktula AM, Grimes HL. Gfi1 regulates miR-21 and miR-196b to control myelopoiesis. Blood. 2009;113(19):47204728.

19. Wang Z, Brandt S, Medeiros A, et al MicroRNA 21 is a homeostatic regulator of macrophage polarization and prevents prostaglandin E2-mediated M2 generation. PLoS One. 2015;10(2):e0115855.

20. Canfran-Duque A, Rotllan N, Zhang X, et al. Macrophage deficiency of miR-21 promotes apoptosis, plaque necrosis, and vascular inflammation during atherogenesis. EMBO Mol Med. 2017;9(9):1244-1262.
21. Lu TX, Lim EJ, Itskovich S, et al. Targeted ablation of miR-21 decreases murine eosinophil progenitor cell growth. PLoS One. 2013;8(3):e59397.

22. Bhagat TD, Zhou L, Sokol L, et al. miR-21 mediates hematopoietic suppression in MDS by activating TGF-beta signaling. Blood. 2013;121(15):2875-2881.

23. Panagal M, S RS, P S, et al. MicroRNA21 and the various types of myeloid leukemia. Cancer Gene Ther. 2018;25(7-8):161-166.

24. Yu Y, Nangia-Makker P, Farhana L, S GR, Levi E, Majumdar AP. miR-21 and miR-145 cooperation in regulation of colon cancer stem cells. Mol Cancer. 2015;14:98.

25. Wu T, Liu Y, Fan Z, et al. miR-21 Modulates the immunoregulatory function of bone marrow mesenchymal stem cells through the PTEN/Akt/TGF-beta1 pathway. Stem Cells. 2015;33(11):3281-3290.

26. Zeng H, Hu M, Lu Y, et al. MicroRNA 34a promotes ionizing radiation-induced DNA damage repair in murine hematopoietic stem cells. FASEB J. 2019;33(7):8138-8147.

27. Petriv OI, Kuchenbauer F, Delaney AD, et al. Comprehensive microRNA expression profiling of the hematopoietic hierarchy. Proc Natl Acad Sci U S A. 2010;107(35):1544315448.

28. Heiser D, Tan YS, Kaplan I, et al. Correlated miR-mRNA expression signatures of mouse hematopoietic stem and progenitor cell subsets predict "stemness" and "myeloid" interaction networks. PLoS One. 2014;9(4): e94852.

29. Venkatraman A, He XC, Thorvaldsen JL, et al. Maternal imprinting at the H19-Igf2 locus maintains adult haematopoietic stem cell quiescence. Nature. 2013;500(7462):345349.

30. Stein SJ, Baldwin AS. Deletion of the NFאB subunit p65/RelA in the hematopoietic compartment leads to defects in hematopoietic stem cell function. Blood. 2013;121(25):5015-5024

31. Mullenders J, Aranda-Orgilles B, Lhoumaud 
$\mathrm{P}$, et al. Cohesin loss alters adult hematopoietic stem cell homeostasis, leading to myeloproliferative neoplasms. J Exp Med. 2015;212(11):1833-1850.

32. Fang J, Muto T, Kleppe M, et al. TRAF6 mediates basal activation of NF-KB necessary for hematopoietic stem cell homeostasis. Cell Rep. 2018;22(5):1250-1262.

33. Shi C, Yang Y, Xia Y, et al. Novel evidence for an oncogenic role of microRNA-21 in colitis-associated colorectal cancer. Gut. 2016;65(9):1470-1481.

34. Wang X, Zhang L, Wei Z, et al. The inhibitory action of PDCD4 in lipopolysaccharide/ D-galactosamine-induced acute liver injury. Lab Invest. 2013;93(3):291-302.

35. Mao XH, Chen M, Wang Y, Cui PG, Liu SB, Xu ZY. MicroRNA-21 regulates the ERK/NF$\kappa \mathrm{B}$ signaling pathway to affect the proliferation, migration, and apoptosis of human melanoma A375 cells by targeting SPRY1, PDCD4, and PTEN. Mol Carcinog. 2017;56(3):886-894.

36. Kraft D, Rall M, Volcic M, et al. NF-kBdependent DNA damage-signaling differentially regulates DNA double-strand break repair mechanisms in immature and mature human hematopoietic cells. Leukemia. 2015;29(7):1543-1554.

37. Burdelya LG, Krivokrysenko VI, Tallant TC, et al. An agonist of toll-like receptor 5 has radioprotective activity in mouse and primate models. Science. 2008;320(5873):226-230.

38. de Laval B, Pawlikowska P, Barbieri D, et al. Thrombopoietin promotes NHEJ DNA repair in hematopoietic stem cells through specific activation of Erk and NF-kB pathways and their target, IEX-1. Blood. 2014;123(4):509-519.

39. Krichevsky AM, Gabriely G. miR-21: a small multi-faceted RNA. J Cell Mol Med. 2009;13(1):39-53.

40. Liu K, Du J, Ruan L. MicroRNA-21 regulate the viability and apoptosis of diffuse large Bcell lymphoma cells by upregulating B cell lymphoma-2. Exp Ther Med. 2017;14(5): 4489-4496.

41. Zhao C, Xiu Y, Ashton J, et al. Noncanonical $\mathrm{NF}-\mathrm{\kappa B}$ signaling regulates hematopoietic stem cell self-renewal and microenvironment interactions. Stem Cells. 2012;30(4) 709-718.

42. Nakagawa MM, Rathinam CV. Constitutive Activation of the canonical NF- $\mathrm{KB}$ pathway leads to bone marrow failure and induction of erythroid signature in hematopoietic stem cells. Cell Rep. 2018;25 (8):2094-2109 e2094.

43. Zhang Z, Zha Y, Hu W, et al. The autoregulatory feedback loop of microRNA-21/programmed cell death protein 4/activation protein-1 (MiR-21/PDCD4/AP-1) as a driv- ing force for hepatic fibrosis development. J Biol Chem. 2013;288(52):37082-37093.

44. Sheedy FI, Palsson-McDermott E, Hennessy EJ, et al. Negative regulation of TLR4 via targeting of the proinflammatory tumor suppressor PDCD4 by the microRNA miR-21. Nat Immunol. 2010;11(2):141-147.

45. Ma X, Becker Buscaglia LE, Barker JR, Li Y MicroRNAs in NF-kappaB signaling. J Mol Cell Biol. 2011;3(3):159-166.

46. Xu G, Wu H, Zhang J, et al. Metformin ameliorates ionizing irradiation-induced longterm hematopoietic stem cell injury in mice. Free Radic Biol Med. 2015;87:15-25.

47. Hu B, Wang X, Hu S, et al. miR-21-mediated Radioresistance occurs via promoting repair of DNA double strand breaks. J Biol Chem. 2017:292(8):3531-3540.

48. Zhang X, Wan G, Berger FG, He X, Lu X The ATM kinase induces microRNA biogenesis in the DNA damage response. Mol Cell. 2011;41(4):371-383

49. Zhu Y, Yu X, Fu H, et al. MicroRNA-21 is involved in ionizing radiation-promoted liver carcinogenesis. Int J Clin Exp Med. 2010;3(3):211-222.

50. Puccetti MV, Adams CM, Dan TD, et al. MicroRNA-21 is required for hematopoietic cell viability after radiation exposure. Int Radiat Oncol Biol Phys. 2019;104(5):11651174 